\title{
The Representation of Scalar Fields with Functions Orthogonal in Polar Coordinates
}

\author{
J. E. Kutzbach and E. W. Warh \\ Universily of Wisconsin, Madison \\ 15 April 1965
}

\section{Introduction}

A variety of analytical methods have been developed over the past 15 years to describe atmospheric flow patterns. Wadsworth (1948) used ortho-normal Tschebycheff polynomials defined in an $x-y$ coordinate system to specify the pressure field over limited latitudelongitude squares. Haurwitz and Craig (1952) suggested the representation of global or hemispheric pressure fields by means of spherical-surface harmonics. Godson (1957) employed Fourier expansions along the latitude circles combined with modified Tschebycheff polynomial expansions along the meridians to describe hemispheric pressure surface topography north of 50N.

Preliminary work has been done at the University of Wisconsin using a Fourier-Bessel expansion to describe hemispheric isobaric topography from $90 \mathrm{~N}$ to $20 \mathrm{~N}$.

\section{Method}

A variable $z$ (here taken to represent the height of an isobaric surface) which is constant on the circumference of the unit circle may be formally expanded in the series

$$
\begin{aligned}
z(r, \lambda)= & \frac{1}{2} \sum_{k=1}^{\infty} A_{o k} J_{0}\left(h_{o k} r\right) \\
& +\sum_{n=1}^{\infty} \sum_{k=1}^{\infty}\left(A_{n k} \cos n \lambda+B_{n k} \sin n \lambda\right) J_{n}\left(h_{n k} r\right),
\end{aligned}
$$

where $r$ and $\lambda$ are polar coordinates, $J_{n}$ is the Bessel function of order $n$, and $h_{n k}$ is the $k$-th positive root of the equation $J_{n}(r)=0$. This expansion will be recognized as the solution to Laplace's equation in polar coordinates.

The functions $J_{n}\left(h_{n k} r\right) \cos n \lambda$ and $J_{n}\left(h_{n k} r\right) \sin n \lambda$ considered for all pairs of subscripts which enter into (1) form an orthogonal set, in the sense that the integral of the product of any two distinct functions of the set over the unit circle in the $r-\lambda$ plane is zero.

The coefficients in (1) are determined from the relations:

$$
\begin{aligned}
& A_{n k}=\frac{1}{\pi d_{n k}} \int_{-\pi}^{\pi} \int_{0}^{1} z(r, \lambda) J_{n}\left(h_{n k} r\right) \cos n \lambda r d r d \lambda, \\
& B_{n k}=\frac{1}{\pi d_{n k}} \int_{-\pi}^{\pi} \int_{0}^{1} z(r, \lambda) J_{n}\left(h_{n k} r\right) \sin n \lambda r d r d \lambda,
\end{aligned}
$$

where $d_{n k}$ denotes the normalizing factor

$$
d_{n k}=\int_{0}^{1} r\left[J_{n}\left(h_{n k} r\right)\right]^{2} d r=\frac{1}{2}\left[J_{n+1}\left(h_{n k}\right)\right]^{2}
$$

Eq. (2) may be written as

$$
\begin{aligned}
A_{n k} & =\frac{1}{d_{n k}} \int_{0}^{1}\left[\frac{1}{\pi} \int_{-\pi}^{\pi} z(r, \lambda) \cos n \lambda d \lambda\right] J_{n}\left(h_{n k} r\right) r d r \\
& =\frac{1}{d_{n k}} \int_{0}^{1}\left[A_{n}(r)\right] J_{n}\left(h_{n k} r\right) r d r .
\end{aligned}
$$

The $A_{n}(r)$ (and $B_{n}(r)$ ) are the familiar Fourier coeffcients.

The variance of the height field about the $r-\lambda$ plane is determined from the sum of the squares of the coefficients and the linear correlation between two fields is determined from the sum of the products of corresponding coefficients. In particular, the portion of the total variance explained by any selected coefficients is given by

$$
E=\sum_{n}^{\prime} \sum_{k}^{\prime}\left(A_{n k}^{*}\right)^{2}+\left(B_{n k}^{*}\right)^{2}
$$

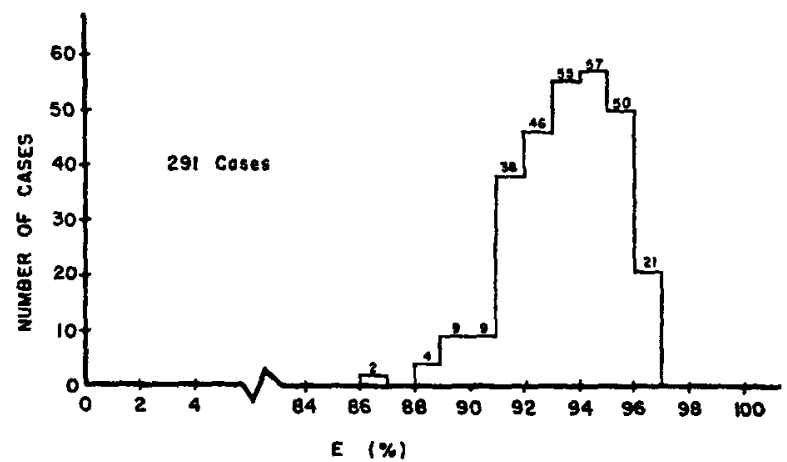

Fic. 1. Frequency distribution of $E$ for 291 cases. $E$ is the percentage of the total variance of the map explained by the 26 Fourier-Bessel coefficients listed below. The 291 cases represent daily $500-\mathrm{mb}$ hemispheric contour maps for the months of January and February, 1959-1963. Fourier-Bessel coefficients used were:

$$
\begin{aligned}
& n=0 \text { (zonal): } \quad A_{01} ; A_{02} ; A_{03} ; A_{04} ; \\
& n=1 \text { (1st harmonic): } A_{11}, B_{11} ; A_{12}, B_{12} ; A_{13}, B_{18} ; \\
& n=2 \text { (2nd harmonic): } A_{21}, B_{21} ; A_{22}, B_{22} ; A_{23}, B_{28} ; \\
& n=3 \text { (3rd harmonic): } A_{21}, B_{21} ; A_{22}, B_{32} ; \\
& n=4 \text { (4th harmonic): } A_{41}, B_{41} ; A_{42}, B_{42} ; \\
& n=5 \text { (5th harmonic): } A_{61}, B_{61} ;
\end{aligned}
$$


where $A^{*}{ }_{n k}$ and $B^{*}{ }_{n k}$ are the normalized values of the Fourier-Bessel coefficients and the primes denote summation over selected values of $n$ and $k$. The linear correlation coefficient between two fields is given by

$$
r_{1,2}=\sum_{n}^{\prime} \sum_{k}^{\prime} A_{n k}{ }^{*}(1) A_{n k}{ }^{*}(2)+B_{n k}{ }^{*}(1) B_{n k}{ }^{*}(2) \text {. }
$$

\section{Results}

To study the efficiency with which the Fourier-Bessel coefficients describe the major features of upper air charts, 222 coefficients ( $A_{n k}, B_{n k}, n=0$ to $18, k=1$ to 6 ) were computed from daily $500-\mathrm{mb}$ data for January and February of 1959 through 1963. During the test period, 34 of the 222 coefficients contributed at least one per cent to the variance of some particular map. (The 222 coefficients explained essentially 100 per cent of the variance of all maps considered.) The coefficient representing the major portion of the zonal flow, $A_{01}$, usually accounted for $60-70$ per cent of the total variance. Certain other coefficients of terms bearing a resemblance to mean atmospheric flow patterns $\left(A_{32}\right.$ and $B_{32}$, for example) also accounted for significant portions of the

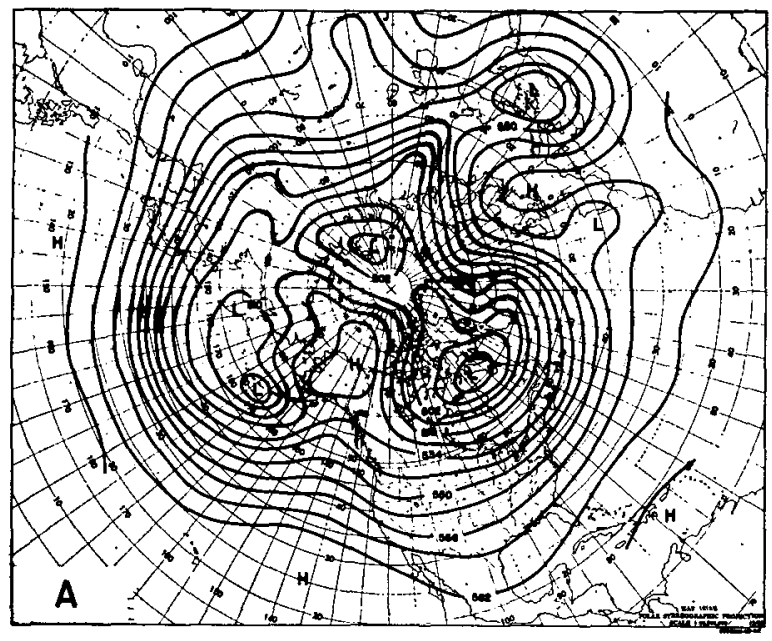

FIG. 2. 500-mb maps for 28 January 1959. $A$ represents the observed map (USWB). $B$ is the reconstructed map (using 26 coefficients). $C$ is the difference between them $(A-B)$. All heights are in tens of meters. total variance. Fig. 1 summarizes the results of the 291 cases studied during the test period.

Programs have been written to permit the reconstruction of the $500-\mathrm{mb}$ map by the addition of selected coefficients. Fig. 2 illustrates the maps for 28 January 1959. Map A shows the observed 500 -mb pattern as obtained from the U. S. Weather Bureau Daily Series Synoptic Weather Maps (Part 1); data for a $10^{\circ}$ by $10^{\circ}$ grid were extracted and reanalyzed. Map B was obtained by recombination of the 26 Fourier-Bessel coefficients listed under Fig. 1 and Map C shows the difference between $\mathrm{A}$ and $\mathrm{B}$. The percentage of the total variance of Map A explained by the 26 coefficients used for the reconstruction of Map B is 96.3 per cent; the remaining root-mean-square error for the entire map is $44 \mathrm{~m}$.

\section{Discussion}

An advantage of this representation over spherical harmonic representation is the elimination of the need for inserting climatological data (which is then heavily weighted) between the lowest latitudes of data coverage
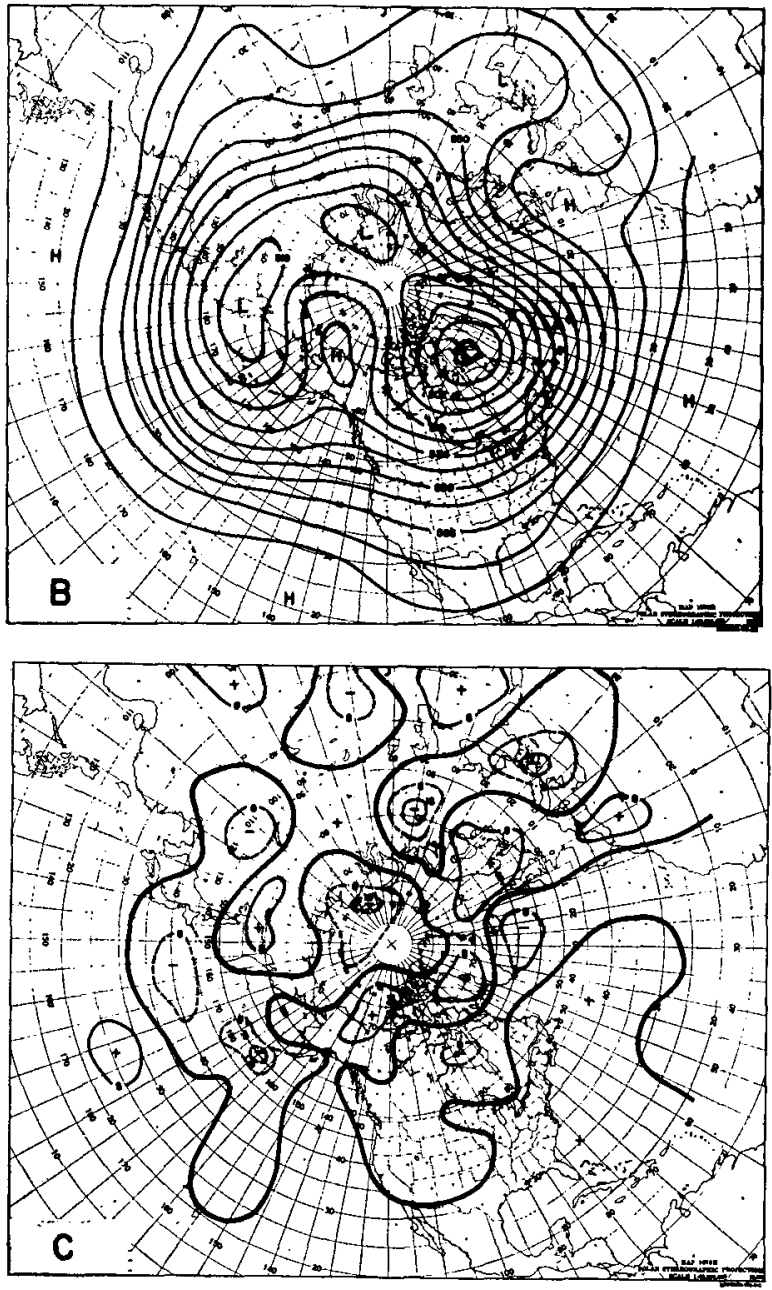
and the equator. Instead, the boundary condition of constant height around the outermost latitude circle is imposed. At $500 \mathrm{mb}$, variations in height around $20 \mathrm{~N}$ are usually less than $50 \mathrm{~m}$. The problem of arbitrary longitudinal limits encountered with Tschebycheff polynomial representations is also removed.

Bryson and Kuhn (1956) have outlined a wide variety of studies involving the use of normalized coefficients of orthogonal functions. This outline is summarized below :

1) Synoptic patterns at different levels or on different dates may be compared.

2) The persistence of certain coefficients or groups of coefficients may be studied using lag correlations or variance spectra.

3) The lag correlations following specified dates or occurences may be examined.

4) Using the geostrophic assumption, the coefficients of the height field for any given level or day can be multiplied by the 1st and 2nd order space derivatives of the orthogonal functions to produce the wind field, the vorticity field, or the kinetic energy field.

5) The coefficients at any two levels may be subtracted to yield the thickness pattern, hence the mean temperature distribution between the two levels.

To this list we can add the following possibility:

6) Zonal harmonic analysis is currently being used to obtain wave number, or scale length estimates of the general circulation parameters (kinetic energy, potential energy, and the conversions between them). The use of a two-dimensional representation allows the study of the area scale of these parameters. The first such computations, using a two-dimensional representation similar to the one described here, have recently been made (Mesinger, 1963).

Acknowledgment. This research was supported in part by the Atmospheric Sciences Division, National Science Foundation under Grant gp-444.

\section{REFERENCES}

Bryson, R. A., and P. M. Kuhn, 1956: Half-hemisphere 500-mb topography description by means of orthogonal polynomials. Scientific Rept. 4, USAF, Contract AF 19(604)-992, University of Wisconsin, Madison.

Godson, W. L., 1957: The numerical representation of atmospheric flow patterns in polar areas. Scientific Rept. 3, Contract AF 19(604)-1141, Arctic Meteor. Res. Group, McGill University, Montreal.

Haurwitz, B., and R. A. Craig, 1952: Atmospheric flow patterns and their representation by spherical-surface harmonics. Geophys. Res. Paper 14, Air Force Cambridge Research Center.

Mesinger, F., 1963: On the two-dimensional spectral analysis of kinetic energy over a circumpolar area of a sphere. Technical Note No. 5, Contract AF 61(052)-366, Institut für Meteor. Technische Hochschule Darmstadt, Darmstadt.

Wadsworth, G. P., J. G. Bryan and C. H. Gordon, 1948: Short range and extended forecasting by statistical methods. Air Weather Service Tech. Rept. 105-37. 